\title{
Ocular Blood Flow and Glaucoma
}

\author{
Lei Fang1, Maneli Mozaffarieh ${ }^{1}$ a \\ ${ }^{1}$ University of Basel, Basel, Switzerland; Limmat Eye Center, Zürich, Switzerland \\ Keywords: glaucoma, ocular blood flow, oxidative stress, metabolic syndrome, Flammer syndrome \\ https://doi.org/10.36000/hbT.2021.02.004
}

healthbook TIMES Das Schweizer Ärztejournal Journal Des Médecins Suisse

Vol. 2, Issue 2, 2021

\begin{abstract}
Glaucoma, a common cause of blindness worldwide, is characterized by an increasing visual field loss and a characteristic excavation of the optic nerve head. Increased intraocular pressure (IOP) is one main risk factor for glaucoma, yet there is increasing evidence that other risk factors are involved in the development of glaucoma, such as impaired ocular blood flow and oxidative stress. ${ }^{1-3}$ On the average, blood flow is reduced in glaucoma patients. It is particularly reduced in patients with normal tension glaucoma as well as in patients with high tension glaucoma who progress despite a normalized IOP. A constant reduction of blood flow as commonly seen in patients with a secondary vascular dysregulation, can lead to atrophy but does not contribute to glaucomatous atrophy. An instability of ocular blood flow however, is clearly linked to occurrence and progression of glaucomatous optic neuropathy. Ocular blood flow is unstable, if IOP fluctuates on a high enough or blood pressure on a low enough level to temporarily exceed the capacity of autoregulatory mechanisms. Ocular blood flow is however also reduced if autoregulation itself is disturbed. Reduced autoregulation occurs particularly in patients suffering from the Flammer-Syndrome. Instability of blood flow (unstable oxygen supply to the optic nerve) leads to oxidative stress, which in turn, leads to the production of peroxynitrite (ONOO-) leading to death of ganglion cells.

This article summarizes the importance of ocular blood flow and oxidative stress for the pathogenesis of glaucoma.
\end{abstract}

\section{Introduction}

Glaucoma, a progressive optic neuropathy, is characterized by a loss of retinal ganglion cells and their axons as well as tissue remodeling that affects both the optic nerve head and the retina. ${ }^{4}$ Clinically, this leads to a visible excavation of the optic nerve and to thinning of the nerve fiber layer of the retina. The patients suffer a progressive loss of the visual field. ${ }^{5}$ Increased intraocular pressure (IOP) is an important risk factor for the development of primary open angle glaucoma. But others risk factors such as impaired ocular blood flow and oxidative stress also play an important role in the pathogenesis of glaucoma.

\section{Oxygen and oxidative stress}

The oxygen molecule is minimally reactive. ${ }^{6}$ This is because the oxygen molecule has two unpaired electrons, each of which are located in a different $\left(\pi^{*}\right)$ antibonding orbitals. These two electrons rotate about their own axis in the same direction and thereby have the same or parallel spins. This is the

\footnotetext{
a Corresponding author:

PD Dr Maneli Mozaffarieh, MD

University of Basel, Basel, Switzerland \& Limmat-Eye Center, Zurich Switzerland

Email: maneli.mozaffarieh@gmail.com
} 
ground state of oxygen which is the most stable state of oxygen. If another atom or molecule would donate two electrons to the oxygen (oxygen gets reduced), both of these electrons would have to be of antiparallel spin, to oxygen's electrons, so as to fit in to the vacant spaces in the $\pi^{*}$ orbitals. ${ }^{7}$ This makes it difficult for the oxygen molecule to accept a pair of electrons from another atomic or molecular orbital, since according to Pauli's principle, a pair of electrons in other atomic or molecular orbitals would have opposite spins. This "spin restriction" forces oxygen to accept its electrons one at a time, explaining why the oxygen molecule reacts sluggishly with many non-radicals. ${ }^{8}$

Oxygen is the most important oxidizing agent in metabolic reactions that lead to the release of energy from the oxidation of various organic molecules. In the respiratory chain (electron transport chain), which takes place in the area of the inner membrane of the mitochondria, the oxygen molecule functions as a terminal electron acceptor. ${ }^{9}$ However, the metabolism of the oxygen molecule does not always work correctly. This malfunction has the consequence that reactive oxygen species ( $\mathrm{ROS}=$ reactive forms of oxygen species) are created. ${ }^{10,11}$

In healthy individuals there is an almost balanced relationship between the production of ROS and the production of antioxidants. If the balance is slightly tipped in favour of the production of ROS there's a need for antioxidant defense and repair as the body can adapt to a certain degree of oxidative stress by enhancing its defense mechanisms. ROS are derived from both endogenous sources (peroxisomes, mitochondria, endoplasmic reticulum, phagocytic cells etc.) as well as from exogenous sources (alcohol, tobacco smoke, pollution, heavy metals, transition metals, industrial solvents, pesticides, certain drugs including paracetamol and radiation). ${ }^{12}$

When the balance, is in favor of the production of ROS then the condition known as oxidative stress is caused (Figure 1). ${ }^{13}$ Oxidative stress causes damage to various cellular macromolecules such as proteins, lipids, sugar residues or DNA; This can potentially lead to cellular growth arrest, cell-related loss of quality, or even cell death. ${ }^{14,15}$ Oxidative stress has been reported to be involved in several diseases such as diabetes mellitus, cardiovascular diseases such as atherosclerosis, respiratory diseases such as asthma, in various cancers and in glaucoma. ${ }^{13,16-18}$

\section{Blood flow in glaucoma}

Most glaucoma patients have impaired blood flow in various tissues of the eye, including the optic nerve, retina, iris and choroid. ${ }^{19}$ In normal pressure glaucoma, the reduction in blood flow is even more pronounced than in high pressure glaucoma. Interestingly, in glaucoma patients, a reduced blood flow is also observed in the capillaries of the fingernail folds. ${ }^{20}$ This suggests that the reduced blood flow is not solely due to an increase in IOP or to glaucoma damage. Rather, there is obviously a primary vascular dysregulation. 


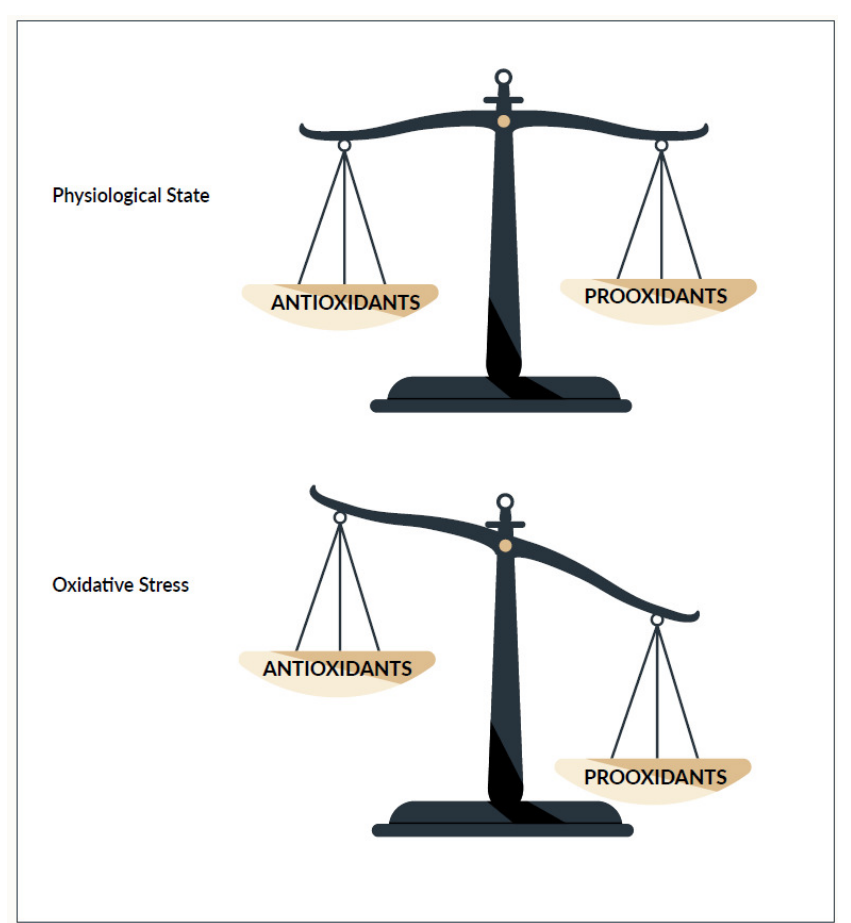

Figure 1. In physiological conditions is a balanced relationship between the production of ROS and the production of antioxidants.

When the balance is in favor of the production of ROS the condition called oxidative stress results. ROS, reactive oxygen species (taken from 4).

Basically, we have two extremes in our population (Figure 2): on the one hand we have patients with high blood pressure, high body mass index, often diabetes type II. They often have a so-called metabolic syndrome (high resistance to insulin). They have a high risk for arteriosclerosis and also a higher probability to develop an increase in IOP and therefore to develop glaucoma, particularly high tension glaucoma.

If we consider the other extreme in the population, we see people with the Flammer Syndrome (FS) with low blood pressure, low body mass index, often cold extremities. There are often physically and intellectually very active. People with FS have a so called primary vascular dysregulation syndrome (PVD), which often interferes with autoregulation of ocular perfusion. ${ }^{2,21}$ For this reason they also have a higher chance to develop glaucoma (particularly normal tension glaucoma (NTG)). This explains why NTG occurs more often in females than in males, in Japanese than in Americans, NTG progresses until a certain age and then often stabilizes.

\section{Primary Vascular dysregulation (Flammer Syndrome)}

Systemic dysregulation can be primary or secondary of nature. ${ }^{23}$ Secondary vascular dysregulation results from other diseases e.g., autoimmune diseases. Secondary vascular dysregulation occurs in the context of diseases in which cells other than the vascular endothelial cells (somewhere in the body) produce an additional amount of endothelin. ${ }^{3}$ Typical examples of diseases are MS, 


\section{Spectrum of our Population}

Flammer-Syndrome

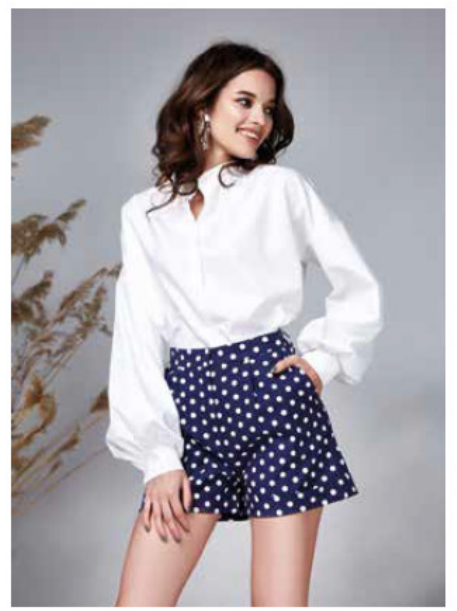

Metabolic-Syndrome

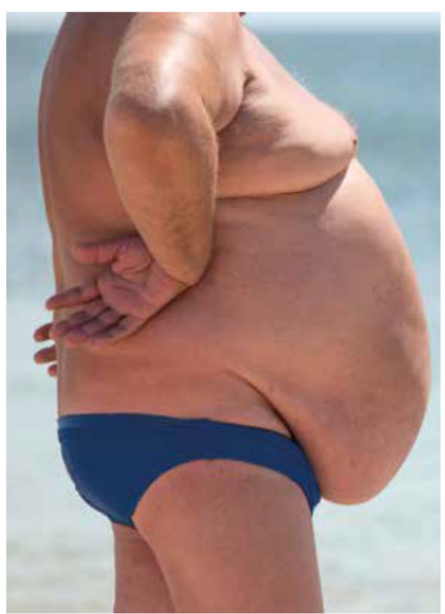

Figure 2. Spectrum of our population.

Whereas on one extreme side of the spectrum of a population we find people with the Flammer Syndrome (low blood pressure, low body mass index, low blood sugar levels), on the other extreme we find those with the metabolic syndrome (high blood pressure, high body mass index, high blood sugar levels). Adapted from Fischer, Mozaffarieh 2018. ${ }^{22}$

arthritis, polyarthritis or AIDS. This leads to a more or less constant reduction of ocular blood flow. It does however, not interfere with autoregulation. Therefore, small vessel disease (SVD) is only a minor risk factor for glaucomatous neuropathy (GON), but a risk factor for some optic nerve head atrophy or even infarction. With primary vascular dysregulation - also known as FS - there is an inborn tendency to react differently to different stimuli such as cold or emotional stress. ${ }^{18}$ In daily practice, people with FS are easy to recognize. These people complain about cold hands and feet (even during the summer months), have a reduced feeling of thirst, need more time to fall asleep, have an altered sense of smell (Figure 3) and often notice a change in sensitivity to drugs and more often have a migraine (www.flammersyndrome.ch).

Patients with FS suffer from a disorder of the autoregulation of their blood circulation, which leads to fluctuations in the IOP or blood pressure values and thus to repeated mild reperfusion damage. ${ }^{16,21}$ This explains why these patients are at an increased risk of developing GON.

In people with FS pulse waves in retinal vessels propagate faster than in people without FS. ${ }^{25}$ This indicates that the rigidity of their retinal vessels in FS is higher despite their anatomically normal appearance. In addition, spatial irregularity of retinal vessels is increased in people with FS, the neurovascular coupling is decreased, and autoregulation of the ocular blood flow is disturbed. ${ }^{26}$ The disturbance of the autoregulation of ocular blood flow 


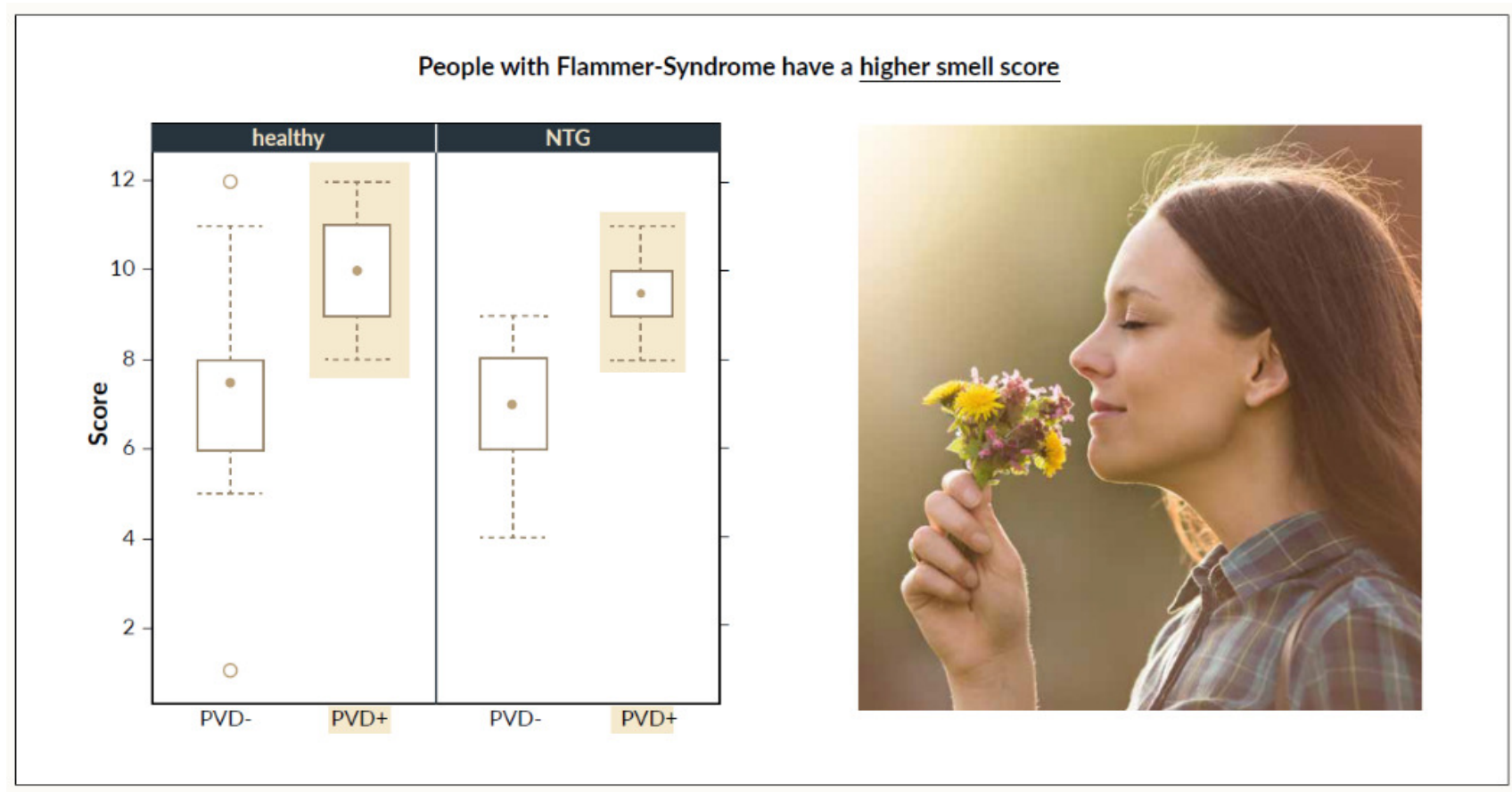

Figure 3. People with Flammer Syndrome can generally smell better than other individuals.

Adapted from Mozaffarieh et al. 2010. ${ }^{24}$

depicts the causal relationship between FS and glaucomatous damage. People with FS also have higher retinal venous pressures as measured by means of ophthalmodynamometry and a higher chance of retinal vein occlusions and central serous chorioretinopathy. ${ }^{27-29}$

At times there's some confusion between the terminology in the use of FS and Raynaud disease. ${ }^{30}$ FS is not the same as a Raynaud disease. Raynaud disease is an autoimmune disease and therefore belongs to the group of SVD. Patients with Raynaud disease do not have a higher risk for glaucoma.

\section{Ocular blood flow disturbances and oxidative stress}

Vascular dysregulation impairs the autoregulation of ocular blood flow. ${ }^{21}$ Physiological or pathologically increased fluctuations in the perfusion pressure therefore also lead to corresponding fluctuations in the ocular blood flow. ${ }^{31,32}$ Repeated mild reperfusion lesions induced by fluctuations in ocular blood flow are part of the actual mechanism causing the damage that leads to GON. Thus, fluctuations in blood flow represent a significantly more serious risk factor for the development of GON than a stable decrease in ocular blood flow. In reperfusion, the blood flow of the optic nerve head, which is particularly subject to fluctuations, increases, and the production of free radicals increases significantly. The area of the optic nerve head is characterized by high energy consumption as the axons there do not have myelin sheaths and therefore contain a large number of mitochondria that produce free radicals as a result of the mild reperfusion damage (especially the superoxide anion). ${ }^{33,34}$ 


\section{Chemical reactions leading to neuronal cell death}

The mitochondria play an important role in reperfusion phenomena. ${ }^{35}$ Mitochondria are the cell's power plants. It is here, where glucose and fat are burned. During this process, the electrons migrate through the respiratory chain from one energy level to the next. In reduced perfusion, this electron transport is partly impaired so the electrons "stack up". As soon as blood flow normalizes again, the electron flow normalizes but because the electrons were previously "stacked up" one single electron may go astray. As soon as this electron encounters an oxygen molecule nearby a free radical (namely the superoxide anion) is formed. ${ }^{15,36}$ An increased formation of free radicals also impairs the reuptake of glutamate. This results in an increase in the extracellular glutamate concentration and thus the phenomenon of excitotoxicity. In the event of excitotoxicity, nitrogen monoxide (NO) is formed increasingly. ${ }^{33,34}$ The latter is also produced when the astrocytes in the retina or optic nerve head are activated. NO is a small, lipophilic molecule that can easily diffuse into neighboring cells, for example into the axons of the optic nerve head. If, as a result of reperfusion, superoxide anion is also present in high concentration, the very harmful peroxynitrite molecule is formed. ${ }^{16}$ Both superoxide anions and peroxynitrite are hydrophilic and therefore cannot diffuse out of the cell through the intact cell membrane. ${ }^{37}$ Superoxide anions and peroxynitrite can, however, diffuse intra-axonally to the retina and to the lateral geniculate body where they cause cell apoptosis.

\section{Discussion}

Glaucoma is a multifactorial disease in which a variety of risk factors such as increased IOP, blood flow disturbances, and oxidative stress play an important role. These factors are interrelated and are involved in a process that ends in damage to the optic nerve and ganglion cells. The disturbance of the ocular blood flow leads to the production of free radicals. These trigger oxidative stress, provided they are not switched off by antioxidant protective mechanisms.

This new interpretation of the pathogenesis of glaucoma is likely to lead to the development of new therapies for the prevention of glaucoma in the future and is also relevant for the early diagnosis of glaucoma. Ocular blood flow and oxidative stress are two crucial parameters in the pathogenesis of this serious eye disease.

\section{Conflict of Interest}

Authors declare no conflict of interest.

\section{Autbor Contributions}

MM took the lead in writing the manuscript. LF provided feedback and made a substantial contribution to the final design of the manuscript. 


\section{Funding}

None.

\section{Acknowledgment}

None.

Submitted: February 07, 2021 CET, Accepted: May 18, 2021 CET licenses/by-nc-sa/4.0 and legal code at https://creativecommons.org/licenses/by-nc-sa/4.0/legalcode for more information. 


\section{REFERENCES}

1. Quigley HA et al. The number of people with glaucoma worldwide in 2010 and 2020. BrJ Ophthalmol.2006;90(3):262-267. doi:10.1136/bjo.2005.081224

2. Flammer J, Mozaffarieh M. What is the present pathogenetic concept of glaucomatous optic neuropathy?. Surv Ophthalmol. 2007;52(Suppl 2):S162-173. doi:10.1016/ j.survophthal.2007.08.012

3. Mozaffarieh $\mathrm{M}$ et al. Is there more to glaucoma treatment than lowering IOP?. Surv Ophthalmol. 2007;52(Suppl 2):S174-179. doi:10.1016/j.survophthal.2007.08.013

4. Mozaffarieh M, Flammer J. Ocular Blood Flow and Glaucomatous Optic Neuropathy. Springer

5. Flammer J et al. Correlation between color vision scores and quantitative perimetry in suspected glaucoma. Arch Ophthalmol. 1984;102(1):38-39. doi:10.1001/archopht.1984.01040030022026

6. Taube H. Mechanisms of oxidation with oxygen.J Gen Physiol. 1965;49(1):S29-52. doi:10.1085/ jgp.49.1.29

7. Halliwell B. Free radicals and antioxidants: a personal view. Nutr Rev. 1994;52(8Pt 1):253-265. doi:10.1111/j.1753-4887.1994.tb01453.x

8. Florez E et al. Theoretical study of the interaction of molecular oxygen with copper clusters. $J$ Phys Chem A. 2005;109(34):7815-7821. doi:10.1021/jp052245

9. Mozaffarieh M et al. Sauerstoff und Durchblutung - zwei wichtige pathogenetische Parameter des Glaukoms. Search of glaucoma. Published online 2007:63-77.

10. Turrens JF. Mitochondrial formation of reactive oxygen species.J Physiol. 2003;552(2):335-344. doi:10.1113/jphysiol.2003.049478

11. Brieger K et al. Reactive oxygen species: from health to disease. Swiss Med Wkly. 2012;142(17):w13659. doi:10.4414/smw.2012.13659

12. Jakubczyk K et al. Reactive oxygen species - sources, functions, oxidative damage. Pol Merkur Lekarski. 2020;48(284):124-127.

13. Mozaffarieh M et al. Oxygen and blood flow: players in the pathogenesis of glaucoma. Mol Vis. 2008;14:224-233.

14. Radi E et al. Apoptosis and oxidative stress in neurodegenerative diseases. J Alzheimers Dis. 2014;42(Suppl 3):S125-152. doi:10.3233/JAD-132738

15. Mozaffarieh $\mathrm{M}$ et al. The potential value of natural antioxidative treatment in glaucoma. Surv Ophthalmol. 2008;53(5):479-505. doi:10.1016/j.survophthal.2008.06.006

16. Mozaffarieh M, Flammer J. New insights in the pathogenesis and treatment of normal tension glaucoma. Curr Opin Pharmacol. 2013;13(1):43-49. doi:10.1016/j.coph.2012.10.001

17. Jomova K, Valko M. Advances in metal-induced oxidative stress and human disease. Toxicology. 2011;283(2-3):65-87. doi:10.1016/j.tox.2011.03.001

18. Vahedian Z et al. Flammer Syndrome and glaucoma. healthbook TIMES Onco Hema. 2020;(1):2-6. doi:10.36000/hbt.2020.01.001

19. Flammer J et al. The mechanism of glaucomatous damage to the optic nerve. Europ Ophthal Rev. 2009;3(1):33-35. doi:10.17925/eor.2009.03.01.33

20. Gasser P et al. Blood-cell velocity in the nailfold capillaries of patients with normal-tension and high-tension glaucoma. Am J Ophthalmol. 1991;111(5):585-588. doi:10.1016/ $\underline{\text { s0002-9394(14)73703-1 }}$

21. Flammer J, Mozaffarieh M. Autoregulation, a balancing act between supply and demand. Can J Ophthalmol. 2008;43(3):317-321. doi:10.3129/i08-056 
22. Fischer J, Mozaffarieh M. Managing Glaucoma beyond Intraocular Pressure. Curr Tre Ophthalmol. 2018;1(1):86-92. doi:10.18314/ctoy.v1i1.1550

23. Flammer J. The vascular concept of glaucoma. Surv Ophthalmol. 1994;38:S3-6. doi:10.1016/ 0039-6257(94)90041-8

24. Mozaffarieh M et al. Smell perception in normal tension glaucoma patients. Mol Vis. 2010;16:506-510.

25. Konieczka K et al. Flammer syndrome. EPMA J. 2014;5(1):11. doi:10.1186/1878-5085-5-11

26. Gherghel $\mathrm{D}$ et al. Is vascular regulation in the central retinal artery altered in persons with vasospasm?. Arch Ophthalmol. 1999;117(10):1359-1362. doi:10.1001/archopht.117.10.1359

27. Fang $L$ et al. The effect of flammer-syndrome on retinal venous pressure. BMC Ophthalmol. 2014;14(1):121. doi:10.1186/1471-2415-14-121

28. Mustur $\mathrm{D}$ et al. Retinal venous pressure measurements in patients with Flammer syndrome and metabolic syndrome. EPMA J. 2017;8(4):339-344. doi:10.1007/s13167-017-0105-7

29. Gauer J et al. Central serous chorioretinopathy in a patient with the Flammer Syndrome. hbT Schw Aerztej. 2021;1:82-84.

30. Mahler F et al. Local cooling test for clinical capillaroscopy in Raynaud's phenomenon, unstable angina, and vasospastic visual disorders. Vasa. 1989;18(3):201-204.

31. Li H, Wu Z. Research advances on relationship between ocular perfusion pressure fluctuations and glaucoma. Zhonghua Yan Ke Za Zhi. 2015;51(6):477-480.

32. Spaniol Ket al. Diurnal Fluctuations of Intraocular Pressure, Blood Pressure, and Ocular Perfusion Pressure in Glaucoma Patients. Klin Monbl Augenheilkd. 2015;232(6):773-778.

33. Haefliger IO, Dettmann E, Liu R, et al. Potential role of nitric oxide and endothelin in the pathogenesis of glaucoma. Surv Ophthalmol. 1999;43(Suppl 1):S51-58. doi:10.1016/ $\underline{\text { s0039-6257(99)00026-0 }}$

34. Neufeld AH et al. Nitric oxide synthase in the human glaucomatous optic nerve head. Arch Ophthalmol. 1997;115(4):497. doi:10.1001/archopht.1997.01100150499009

35. Szeto HH. Mitochondria-targeted cytoprotective peptides for ischemia-reperfusion injury. Antioxid Redox Signal. 2008;10(3):601-619. doi:10.1089/ars.2007.1892

36. Chouchani ET, Pell VR, James AM, et al. A Unifying Mechanism for Mitochondrial Superoxide Production during Ischemia-Reperfusion Injury. Cell Metab. 2016;23(2):254-263. doi:10.1016/j.cmet.2015.12.009

37. Ferrer-Sueta G, Radi R. Chemical biology of peroxynitrite: kinetics, diffusion, and radicals. ACS Chem Biol. 2009;4(3):161-177. doi:10.1021/cb800279q 